\title{
Periodic solutions of semilinear wave equations with discontinuous nonlinearities
}

\author{
In-Sook Kim*, Jung-Hyun Bae and Suk-Joon Hong
}

\author{
"Correspondence: iskim@skku.edu \\ Department of Mathematics, \\ Sungkyunkwan University, Suwon, \\ 440-746, Republic of Korea
}

\begin{abstract}
We study the periodic Dirichlet problem for a semilinear wave equation with discontinuous nonlinearity. First we establish a continuation theorem for a semilinear operator equation in a Hilbert space, where a key tool is the Berkovits-Tienari degree theory for a class of perturbations of monotone type of a densely defined closed linear operator. Applying the continuation theorem, we prove the main results on the solvability of the given semilinear wave equation, with the aid of spectral theory for densely defined closed linear operators.
\end{abstract}

MSC: 35L05; 35R05; 47H04; 47H05; 47H11

Keywords: semilinear wave equation; multi-valued operator; pseudomonotone operator; degree theory

\section{Introduction}

The study of nonlinear wave equations has been developed in various ways of approach by many researchers; for instance, by Brézis and Nirenberg [1, 2], Rabinowitz [3], Berkovits and Mustonen [4-6]. To find periodic solutions of a nonlinear wave equation, Mawhin and Willem [7] established a Leray-Schauder type continuation theorem for abstract equations involving some perturbations of monotone type of a linear operator in a Hilbert space, where the Galerkin approximation method was used; see also [8]. In this aspect, Berkovits and Mustonen [9] showed the existence of weak solutions of the periodic Dirichlet problem for a semilinear wave equation under various conditions on the nonlinearity. Moreover, Berkovits and Tienari [10] constructed a topological degree theory for some classes of multi-valued operators of monotone type with elliptic super-regularization method, with applications to hyperbolic problems with discontinuous nonlinearity.

In this paper, we study a semilinear wave equation of the form

$$
\begin{cases}u_{t t}-u_{x x}-h(x, t) \in-[\underline{g}(x, t, u), \bar{g}(x, t, u)] & \text { for almost all }(x, t) \in(0, \pi) \times \mathbb{R} \\ u(0, t)=u(\pi, t)=0 & \text { for all } t \in \mathbb{R} \\ u \text { is } 2 \pi \text {-periodic in } t, & \end{cases}
$$

where $g:[0, \pi] \times \mathbb{R} \times \mathbb{R} \rightarrow \mathbb{R}$ is a possibly discontinuous function in the third variable to be specified later. To seek a weak solution of the problem (1.1), we consider the corresponding

(c) 2015 Kim et al. This article is distributed under the terms of the Creative Commons Attribution 4.0 International License (http://creativecommons.org/licenses/by/4.0/), which permits unrestricted use, distribution, and reproduction in any medium, provided you give appropriate credit to the original author(s) and the source, provide a link to the Creative Commons license, and indicate if changes were made. 
semilinear equation

$$
h \in L u+N u
$$

in a Hilbert space $L^{2}(\Omega)$, where $L$ is a densely defined closed linear operator with an infinite dimensional kernel and $N$ is a bounded upper semicontinuous multi-valued operator of monotone type.

Using the Berkovits-Tienari degree theory for operators of the type $L+N$, we first establish a continuation theorem for the semilinear equation (1.2), connecting to a reference map having nonzero degree. Actually, the linear operator $L$ modeled by the differential operator $\partial^{2} / \partial t^{2}-\partial^{2} / \partial x^{2}$ and the Nemytskii operator $N$ generated by certain nonlinearity $g$, have the desired properties for the use of the topological degree; see $[8,11,12]$.

Next, we prove our main results on the solvability of the problem (1.1), by applying the continuation theorem to the reference maps $L+P$ and $L+c I$, where $P$ denotes the orthogonal projection onto the kernel of $L, I$ denotes the identity operator, and $-c$ is a positive regular value of $L$. Thus, spectral theory for densely defined closed linear operators plays a decisive role in the study of semilinear equations. Analogous and related results were given in $[9,10]$; see also $[7,8]$.

\section{Continuation theorem}

Let $H$ be a real Hilbert space with an inner product $(\cdot, \cdot)$. Given a nonempty subset $\Omega$ of $H$, let $\bar{\Omega}$ and $\partial \Omega$ denote the closure and the boundary of $\Omega$ in $H$, respectively. Let $B_{r}(u)$ denote the open ball in $H$ of radius $r>0$ centered at $u$. The symbol $\rightarrow(\rightarrow)$ stands for strong (weak) convergence.

Definition 2.1 A multi-valued operator $F: \Omega \subset H \rightarrow 2^{H}$ is said to be:

(1) upper semicontinuous if the set $F^{-1}(A)=\{u \in \Omega \mid F u \cap A \neq \emptyset\}$ is closed for all closed sets $A$ in $H$;

(2) weakly upper semicontinuous if $F^{-1}(A)$ is closed for all weakly closed sets $A$ in $H$;

(3) bounded if it maps bounded sets into bounded sets;

(4) compact if it is upper semicontinuous and the image of any bounded set is relatively compact.

Definition 2.2 A multi-valued operator $F: \Omega \subset H \rightarrow 2^{H} \backslash \emptyset$ is said to be:

(1) of class $\left(S_{+}\right)$if for any sequence $\left(u_{n}\right)$ in $\Omega$ and for any sequence $\left(w_{n}\right)$ in $H$ with $w_{n} \in F u_{n}$ such that $u_{n} \rightarrow u$ in $H$ and

$$
\limsup _{n \rightarrow \infty}\left(w_{n}, u_{n}-u\right) \leq 0,
$$

we have $u_{n} \rightarrow u$ in $H$;

(2) pseudomonotone if for any sequence $\left(u_{n}\right)$ in $\Omega$ and for any sequence $\left(w_{n}\right)$ in $H$ with $w_{n} \in F u_{n}$ such that $u_{n} \rightarrow u$ in $H$ and

$$
\limsup _{n \rightarrow \infty}\left(w_{n}, u_{n}-u\right) \leq 0,
$$

we have $\lim _{n \rightarrow \infty}\left(w_{n}, u_{n}-u\right)=0$ and if $u \in \bar{\Omega}$ and $w_{j} \rightarrow w$ in $H$ for some subsequence $\left(w_{j}\right)$ of $\left(w_{n}\right)$, then $w \in F u$; 
(3) quasimonotone if for any sequence $\left(u_{n}\right)$ in $\Omega$ and for any sequence $\left(w_{n}\right)$ in $H$ with $w_{n} \in F u_{n}$ such that $u_{n} \rightarrow u$ in $H$, we have

$$
\liminf _{n \rightarrow \infty}\left(w_{n}, u_{n}-u\right) \geq 0
$$

(4) monotone if

$$
(w-y, u-v) \geq 0 \quad \text { for all } u, v \in \Omega, w \in F u \text { and } y \in F v \text {. }
$$

Note that the class of $\left(S_{+}\right)$-operators is invariant under quasimonotone perturbations and each pseudomonotone bounded operator is quasimonotone. Moreover, if $F: H \rightarrow 2^{H}$ is monotone, then it is pseudomonotone.

Let $(H,(\cdot, \cdot))$ be a real separable Hilbert space. Suppose that $L: D(L) \subset H \rightarrow H$ is a densely defined closed linear operator with

$$
\operatorname{Im} L=(\operatorname{Ker} L)^{\perp}
$$

and $K: \operatorname{Im} L \rightarrow \operatorname{Im} L$, the inverse of the restriction of $L$ to $\operatorname{Im} L \cap D(L)$, is compact. Let $P$ and $Q$ be the orthogonal projections onto $\operatorname{Ker} L$ and $\operatorname{Im} L$, respectively.

In what follows, we always assume that all multi-valued operators considered have nonempty closed convex values.

For any open set $G$ in $H$, let

$$
\begin{aligned}
\mathcal{F}_{G}:= & \left\{L+N \mid N: \bar{G} \rightarrow 2^{H}\right. \text { is bounded, weakly upper semicontinuous, and } \\
& \left.P N=P \hat{N} \text { for some }\left(S_{+}\right) \text {-operator } \hat{N}: \bar{G} \rightarrow 2^{H}\right\} .
\end{aligned}
$$

Following Berkovits and Tienari [10], one can define a topological degree function for the class $\mathcal{F}_{G}$.

Definition 2.3 Let $G$ be a bounded open set in $H$ and $L+N \in \mathcal{F}_{G}$. If $h \notin(L+N)(\partial G \cap$ $D(L)$ ), then the degree $d$ is defined in terms of the Leray-Schauder degree $d_{\text {LS }}$ as follows:

$$
d(L+N, G, h):=\lim _{\lambda \rightarrow \infty} d_{\mathrm{LS}}\left(F_{\lambda}, G, h_{\lambda}\right)
$$

where $F_{\lambda}=I+\left(K Q+\lambda \Psi^{2} P\right) N$ and $h_{\lambda}=\left(K Q+\lambda \Psi^{2} P\right) h$. Here $\Psi: \operatorname{Ker} L \rightarrow \operatorname{Ker} L$ is a compact self-adjoint linear injection.

The degree function $d$ defined above satisfies the usual basic properties, as in [10].

Lemma 2.4 Suppose that $G$ is any bounded open subset of $X, L+N \in \mathcal{F}_{G}$, and $h \notin(L+$ $N)(\partial G \cap D(L))$. Then the degree $d$ has the following properties:

(a) (Existence) If $d(L+N, G, h) \neq 0$, then $h \in(L+N)(G \cap D(L))$.

(b) (Additivity) If $G_{1}$ and $G_{2}$ are disjoint open subsets of $G$ such that $h \notin(L+N)\left(\bar{G} \backslash\left(G_{1} \cup G_{2}\right)\right)$, then we have

$$
d(L+N, G, h)=d\left(L+N, G_{1}, h\right)+d\left(L+N, G_{2}, h\right) .
$$


(c) (Homotopy invariance) Suppose that $N, \hat{N}:[0,1] \times \bar{G} \rightarrow 2^{H}$ are bounded weakly upper semicontinuous homotopies with nonempty closed convex values such that $\hat{N}$ is of class $\left(S_{+}\right)$and $P N=P \hat{N}$. If $h:[0,1] \rightarrow H$ is a continuous path in $H$ such that

$$
h(t) \notin(L+N(t, \cdot))(\partial G \cap D(L)) \text { for all } t \in[0,1],
$$

then $d(L+N(t, \cdot), G, h(t))$ is constant for all $t \in[0,1]$.

(d) (Normalization) If $h \in(L+P)(G \cap D(L))$, then we have $d(L+P, G, h)=1$.

Here, the homotopy $\hat{N}$ is of class $\left(S_{+}\right)$in the sense that for any sequence $\left(t_{n}, u_{n}\right)$ in $[0,1] \times$ $\bar{G}$ and for any sequence $\left(a_{n}\right)$ in $H$ with $a_{n} \in \hat{N}\left(t_{n}, u_{n}\right)$ such that $u_{n} \rightarrow u$ in $H$ and

$$
\limsup _{n \rightarrow \infty}\left(a_{n}, u_{n}-u\right) \leq 0
$$

we have $u_{n} \rightarrow u$ in $H$.

Definition 2.5 A single-valued operator $L+N_{0} \in \mathcal{F}_{H}$ is called a reference map if it is injective and $d\left(L+N_{0}, G, h\right) \neq 0$ for any bounded open set $G \subset H$ with $h \in\left(L+N_{0}\right)(G \cap$ $D(L))$.

The typical examples of a reference map are $L+P, L+c I$ with $-c \notin \sigma(L)$, and $L+c I+P_{c}$ with $-c \in \sigma(L)$, where $c>0$ and $P_{c}$ denotes the orthogonal projection onto $\operatorname{Ker}(L+c I)$; see [9].

As a key tool, we need the following continuation result on pseudomonotone operators, which follows from the homotopy invariance of the above degree. For the single-valued case, we refer to Theorem 7 of [9].

Theorem 2.6 Let $G$ be a bounded open set in $H$ with $0 \in G$. Suppose that $L+N_{0} \in \mathcal{F}_{H}$ is a reference map with $0 \in\left(L+N_{0}\right)(G \cap D(L))$ and $N: \bar{G} \rightarrow 2^{H}$ is a pseudomonotone bounded weakly upper semicontinuous operator. If the following boundary condition holds for a given $h \in H$ :

$$
\text { th } \notin L u+t N u+(1-t) N_{0} u \quad \text { for all } u \in \partial G \cap D(L) \text { and all } t \in[0,1),
$$

then the equation $h \in(L+N)$ u has a solution in $\bar{G} \cap D(L)$.

Proof If $h \in(L+N)(\partial G \cap D(L))$, there is nothing to prove. We now suppose that $h \notin$ $(L+N)(\partial G \cap D(L))$. If the condition (2.1) holds for the case $L+N \in \mathcal{F}_{G}$, Lemma 2.4 and Definition 2.5 imply that

$$
d(L+N, G, h)=d\left(L+N_{0}, G, 0\right) \neq 0 .
$$

To prove our pseudomonotone case, we set

$$
N_{\varepsilon}=N+\varepsilon N_{0} \quad \text { for each } \varepsilon>0 .
$$


It is clear that $L+N_{\varepsilon} \in \mathcal{F}_{G}$. Notice that $(L+N)(\bar{G} \cap D(L))$ is closed in $H$ since the graph of $L$ is weakly closed and $N$ is pseudomonotone. Indeed, let $\left(u_{n}\right)$ be a sequence in $\bar{G} \cap D(L)$ such that

$$
L u_{n}+a_{n} \rightarrow y \quad \text { in } H
$$

where $a_{n} \in N u_{n}$. Without loss of generality, we may suppose that $u_{n} \rightarrow u$ in $H$ and $a_{n} \rightarrow a$ in $H$. Since the graph of $L$ is weakly closed, we have $u \in D(L)$ and $L u=y-a$. Since the operator $K$ is compact, the sequence $\left(u_{n}\right)$ has a convergent subsequence $\left(u_{n_{k}}\right)$, say $u_{n_{k}} \rightarrow v$ for some $v \in \bar{G}$. By the uniqueness of weak limit, we have $u=v \in \bar{G}$. Since the operator $N$ is pseudomonotone, $u_{n_{k}} \rightarrow u$ and $a_{n_{k}} \rightarrow a$ imply $a \in N u$. Therefore, we get

$$
y=L u+a \in(L+N)(\bar{G} \cap D(L)) .
$$

We have just shown that $(L+N)(\bar{G} \cap D(L))$ is closed in $H$.

Now there are two cases to consider. First, we assume that there exists a positive number $\varepsilon_{0}$ such that the following condition holds for every $\varepsilon \in\left(0, \varepsilon_{0}\right)$ :

$$
\text { th } \notin L u+t N_{\varepsilon} u+(1-t) N_{0} u \quad \text { for all } u \in \partial G \cap D(L) \text { and all } t \in[0,1) .
$$

Applying (2.2) with $N_{\varepsilon}$ instead of $N$, we get

$$
d\left(L+N_{\varepsilon}, G, h\right) \neq 0 \quad \text { for every } \varepsilon \in\left(0, \varepsilon_{0}\right) .
$$

Let $\left(\varepsilon_{n}\right)$ be a sequence in $\left(0, \varepsilon_{0}\right)$ with $\varepsilon_{n} \rightarrow 0$. In view of Lemma 2.4, let $\left(u_{n}\right)$ be a sequence in $G \cap D(L)$ such that

$$
L u_{n}+a_{n}+\varepsilon_{n} N_{0} u_{n}=h \quad \text { for all } n \in \mathbb{N},
$$

where $a_{n} \in N u_{n}$. Then we have $L u_{n}+a_{n} \rightarrow h$. Since $(L+N)(\bar{G} \cap D(L))$ is closed in $H$, this implies that

$$
h \in \overline{(L+N)(G \cap D(L))} \subset \overline{(L+N)(\bar{G} \cap D(L))}=(L+N)(\bar{G} \cap D(L)) .
$$

Next, we assume that there are sequences $\left(\varepsilon_{n}\right)$ in $(0, \infty)$ with $\varepsilon_{n} \rightarrow 0,\left(t_{n}\right)$ in $[0,1),\left(u_{n}\right)$ in $\partial G \cap D(L)$, and $\left(a_{n}\right)$ with $a_{n} \in N u_{n}$ such that

$$
t_{n} h=L u_{n}+t_{n}\left(a_{n}+\varepsilon_{n} N_{0} u_{n}\right)+\left(1-t_{n}\right) N_{0} u_{n} \quad \text { for all } n \in \mathbb{N} \text {. }
$$

Passing to subsequences if necessary, we may suppose that

$$
t_{n} \rightarrow t \in[0,1], \quad u_{n} \rightarrow u \quad \text { in } H \quad \text { and } \quad a_{n} \rightarrow a \text { in } H .
$$

Taking the transformation $Q K Q$ at both sides of (2.3), we get $Q u_{n} \rightarrow Q u$, by the compactness of the operator $K$. Actually, we have $t=1$. In fact, assume that $t \neq 1$. Since $P N_{0}=P \hat{N}_{0}$ 
for some bounded $\left(S_{+}\right)$-operator $\hat{N}_{0}$ and $N$ is bounded and quasimonotone, we have by (2.3)

$$
\begin{aligned}
\limsup _{n \rightarrow \infty}\left(\hat{N}_{0} u_{n}, u_{n}-u\right) & =\limsup _{n \rightarrow \infty}\left(\hat{N}_{0} u_{n}, P u_{n}-P u\right) \\
& =\limsup _{n \rightarrow \infty}\left(P N_{0} u_{n}, u_{n}-u\right) \\
& =\limsup _{n \rightarrow \infty}\left[-\frac{t_{n}}{1-t_{n}+t_{n} \varepsilon_{n}}\left(P a_{n}-P h, u_{n}-u\right)\right] \\
& =-\frac{t}{1-t} \liminf _{n \rightarrow \infty}\left(a_{n}, u_{n}-u\right) \\
& \leq 0 .
\end{aligned}
$$

Since $\hat{N}_{0}$ is of class $\left(S_{+}\right)$and $N$ is pseudomonotone, we obtain $u_{n} \rightarrow u \in \partial G$ and $a \in N u$. Moreover, since the graph of $L$ is weakly closed, we obtain from (2.3) that

$$
u \in D(L) \text { and } \quad L u=t h-t a-(1-t) N_{0} u, \quad \text { that is, } \quad t h \in L u+t N u+(1-t) N_{0} u \text {, }
$$

which contradicts the hypothesis (2.1). Thus, we have just shown that $t=1$. Therefore, it follows from (2.3) that $L u_{n}+a_{n} \rightarrow h$, which implies as above

$$
h \in \overline{(L+N)(\partial G \cap D(L))} \subset(L+N)(\bar{G} \cap D(L)) .
$$

In all possible cases, we conclude that $h \in(L+N)(\bar{G} \cap D(L))$. This completes the proof.

Remark 2.7 We point out that in Theorem 2.6 the convexity assumption on the open set $G$ can be relaxed, in comparison to Theorem 7 of [9]. In fact, we did not need the weak closedness of the set $G$ in the whole proof.

\section{Main results}

This section is devoted to the solvability of a semilinear wave equation with discontinuous nonlinearity, based on the continuation theorem on pseudomonotone operators stated in the previous section.

We consider the semilinear wave equation of the form

$$
\begin{cases}u_{t t}-u_{x x}-h(x, t) \in-[\underline{g}(x, t, u), \bar{g}(x, t, u)] & \text { for almost all }(x, t) \in(0, \pi) \times \mathbb{R} \\ u(0, t)=u(\pi, t)=0 & \text { for all } t \in \mathbb{R}, \\ u \text { is } 2 \pi \text {-periodic in } t, & \end{cases}
$$

where

$$
\underline{g}(x, t, s)=\liminf _{\eta \rightarrow s} g(x, t, \eta) \quad \text { and } \quad \bar{g}(x, t, s)=\limsup _{\eta \rightarrow s} g(x, t, \eta) .
$$

Let $\Omega=(0, \pi) \times(0,2 \pi)$ and let $H=L^{2}(\Omega)$ be the real Hilbert space with usual inner product $(\cdot, \cdot)$ and norm $\|\cdot\|$. Let $\varphi_{n m}(x, t)=\pi^{-1} \sin (n x) \exp (i m t)$ for $n \in \mathbb{N}$ and $m \in \mathbb{Z}$. Each 
$u \in H$ has a representation

$$
u=\sum_{n \in \mathbb{N}, m \in \mathbb{Z}} u_{n m} \varphi_{n m}
$$

where $u_{n m}=\left(u, \varphi_{n m}\right)$.

We define a linear operator $L: D(L) \subset H \rightarrow H$ by

$$
L u=\sum_{n \in \mathbb{N}, m \in \mathbb{Z}}\left(n^{2}-m^{2}\right) u_{n m} \varphi_{n m}
$$

where

$$
D(L)=\left\{u \in H: \sum_{n \in \mathbb{N}, m \in \mathbb{Z}}\left|n^{2}-m^{2}\right|^{2}\left|u_{n m}\right|^{2}<\infty\right\} .
$$

Then $D(L)$ is a dense subspace of $H$ and $L$ is a self-adjoint operator. Moreover, we have $\operatorname{Ker} L=\operatorname{span}\left\{\varphi_{n n}, \varphi_{n,-n}: n \in \mathbb{N}\right\}, \operatorname{Im} L=(\operatorname{Ker} L)^{\perp}$ and $L$ has a pure point spectrum $\sigma(L)$ of isolated eigenvalues:

$$
\sigma(L)=\left\{n^{2}-m^{2}: n \in \mathbb{N}, m \in \mathbb{Z}\right\} .
$$

Let $L_{0}$ be the restriction of $L$ to the subspace $\operatorname{Im} L$. Then the operator $L_{0}: \operatorname{Im} L \cap D(L) \rightarrow$ $\operatorname{Im} L$ is a bijection with the inverse $L_{0}^{-1}: \operatorname{Im} L \rightarrow \operatorname{Im} L$ given by

$$
L_{0}^{-1} u=\sum_{(n, m) \in \Gamma}\left(n^{2}-m^{2}\right)^{-1} u_{n m} \varphi_{n m}
$$

where $\Gamma=\left\{(n, m) \in \mathbb{N} \times \mathbb{Z}: n^{2} \neq m^{2}\right\}$. Note that the spectrum of the bounded inverse operator $L_{0}^{-1}$ has no limit point except 0 and $\operatorname{dim} \operatorname{Ker}\left(L_{0}^{-1}-\lambda I\right)$ is finite for every $\lambda \in \sigma\left(L_{0}^{-1}\right)$ with $\lambda \neq 0$. This implies that $L_{0}^{-1}$ is compact. See e.g., [7, 12].

Suppose that $g: \Omega \times \mathbb{R} \times \mathbb{R} \rightarrow \mathbb{R}$ is $2 \pi$-periodic in the second variable $t$ such that

(g1) $\bar{g}$ and $\underline{g}$ are superpositionally measurable, that is, $\bar{g}(\cdot, \cdot, u(\cdot, \cdot))$ and $\underline{g}(\cdot, \cdot, u(\cdot, \cdot))$ are measurable on $\Omega$ for any measurable function $u: \Omega \rightarrow \mathbb{R}$;

(g2) $g$ satisfies the growth condition:

$$
|g(x, t, s)| \leq \theta|s|+h_{0}(x, t) \quad \text { for almost all }(x, t) \in \Omega \text { and all } s \in \mathbb{R},
$$

where $\theta$ is a positive constant and $h_{0} \in H$ is nonnegative;

(g3) $g$ is nondecreasing in the third variable $s$, that is,

$$
(g(x, t, s)-g(x, t, \eta))(s-\eta) \geq 0 \quad \text { for almost all }(x, t) \in \Omega \text { and all } s, \eta \in \mathbb{R} .
$$

We define a multi-valued operator $N: H \rightarrow 2^{H}$ by setting

$$
N u=\{w \in H \mid \underline{g}(x, t, u(x, t)) \leq w(x, t) \leq \bar{g}(x, t, u(x, t)) \text { for almost all }(x, t) \in \Omega\} .
$$

Under assumptions (g1) and (g2), the multi-valued operator $N$ is bounded, upper semicontinuous, and $N u$ is nonempty, closed, and convex for every $u \in H$; see Theorem 1.1 
of [11]. Under additional assumption (g3), the operator $N$ is monotone and hence pseudomonotone.

Definition 3.1 A point $u \in H$ is said to be a weak solution of the problem (3.1) if there exists a point $w \in N u$ such that

$$
\left(u, v_{t t}-v_{x x}\right)+(w, v)=(h, v) \quad \text { for all } v \in C^{2},
$$

where $C^{2}$ denotes the space of twice continuously differentiable functions $v: \bar{\Omega} \rightarrow \mathbb{R}$ such that

$$
v(0, \cdot)=v(\pi, \cdot)=0 \quad \text { and } \quad v(\cdot, 0)-v(\cdot, 2 \pi)=v_{t}(\cdot, 0)-v_{t}(\cdot, 2 \pi)=0 .
$$

In view of (3.2) and (3.3), $u \in H$ is a weak solution of the problem (3.1) if and only if $u \in D(L)$ is a solution of the operator equation

$$
h \in L u+N u \text {. }
$$

Notice that $\lambda_{0}=3$ is the first positive eigenvalue of $-L$ and

$$
(L u, u) \geq-\frac{1}{3}\|L u\|^{2} \quad \text { for all } u \in D(L) .
$$

For our aim, we first present a multi-valued version of Proposition A.6 of [1]. For this, we need the Cauchy inequality for every positive number $\varepsilon$ :

$$
a b \leq \varepsilon a^{2}+\frac{b^{2}}{4 \varepsilon} \quad \text { for all positive numbers } a \text { and } b .
$$

Lemma 3.2 Suppose that $g: \Omega \times \mathbb{R} \times \mathbb{R} \rightarrow \mathbb{R}$ satisfies the conditions (g1), (g3) and the growth condition (g2) with $\theta<3$. Then there exists a positive constant $\gamma$ with $\theta<\gamma<3$ such that the following relation holds for any given $h, v \in H$ with $h \in N v$ :

$$
(w-h, u) \geq \frac{1}{\gamma}\|w\|^{2}-C(h, v) \quad \text { for all } u \in H \text { and } w \in N u,
$$

where $C(h, v)$ is a positive constant that depends on $h$ and $v$.

Proof Let $h, v \in H$ with $h \in N v$ be given. Note by (g3) that $N$ is monotone. For all $u \in H$ and $w \in N u$, using the growth condition (g2) and the Hölder inequality gives

$$
\begin{aligned}
(w-h, u) & \geq \int_{\Omega}|w-h||u-v|-\int_{\Omega}|w-h||v| \\
& \geq \int_{\Omega}(|w-h|)\left(\frac{|w|-h_{0}}{\theta}-2|v|\right) \\
& \geq \int_{\Omega}(|w|-|h|) \frac{|w|}{\theta}-(|w|+|h|)\left(\frac{h_{0}}{\theta}+2|v|\right) \\
& \geq \frac{1}{\theta}\|w\|^{2}-\|w\|\left(\frac{\|h\|+\left\|h_{0}\right\|+2 \theta\|v\|}{\theta}\right)-\|h\|\left(\frac{\left\|h_{0}\right\|+2 \theta\|v\|}{\theta}\right) .
\end{aligned}
$$


Applying the Cauchy inequality (3.6) with $\varepsilon=1 / 2(1 / \theta-1 / 3)$ and $a=\|w\|$, we obtain

$$
\begin{aligned}
(w-h, u) \geq & \left(\frac{1}{\theta}-\varepsilon\right)\|w\|^{2}-\frac{1}{4 \varepsilon \theta^{2}}\left(\|h\|+\left\|h_{0}\right\|+2 \theta\|v\|\right)^{2} \\
& -\|h\|\left(\frac{\left\|h_{0}\right\|+2 \theta\|v\|}{\theta}\right)
\end{aligned}
$$

Setting $\gamma:=[1 / 2(1 / \theta+1 / 3)]^{-1}$, it follows from (3.7) that $\theta<\gamma<3$ and

$$
(w-h, u) \geq \frac{1}{\gamma}\|w\|^{2}-C(h, v) \quad \text { for all } u \in H \text { and } w \in N u,
$$

where $C(h, v)$ denotes a positive constant depending on $h$ and $v$. This completes the proof.

Now we prove that (3.1) has a weak solution, based on the use of reference map $L+P$. We give a substantially simpler and more direct proof in a precise manner, in comparison to Corollary 5.2 of [10].

Theorem 3.3 Suppose that $g: \Omega \times \mathbb{R} \times \mathbb{R} \rightarrow \mathbb{R}$ satisfies the conditions (g1), (g3) and the growth condition (g2) with $\theta<3$. Further, suppose that there is a positive constant $\eta$ and $a$ measurable function $h_{1} \in H$ such that

$$
|g(x, t, s)| \geq \eta|s|-h_{1}(x, t) \quad \text { for almost all }(x, t) \in \Omega \text { and all } s \in \mathbb{R}
$$

Then (3.1) has a weak solution for each $h \in H$.

Proof Let $h \in H$ be arbitrary but fixed. It follows from condition (3.8) that

$$
\|w\| \geq \eta\|u\|-\left\|h_{1}\right\| \quad \text { for all } u \in H \text { and all } w \in N u \text {. }
$$

Then the monotone operator $N$ is surjective and so there exists a point $v \in H$ such that $h \in N v$. We first show that there is a positive constant $R$ such that

$$
0 \notin L u+t(N u-h)+(1-t) P u \quad \text { for all } u \in D(L) \text { with }\|u\|=R \text { and all } t \in(0,1) \text {. }
$$

In fact, assume to the contrary that there are sequences $\left(u_{n}\right)$ in $D(L)$ with $\left\|u_{n}\right\| \rightarrow \infty,\left(t_{n}\right)$ in the open interval $(0,1)$ with $t_{n} \rightarrow t$, and $\left(w_{n}\right)$ in $H$ with $w_{n} \in N u_{n}$ such that

$$
0=L u_{n}+t_{n}\left(w_{n}-h\right)+\left(1-t_{n}\right) P u_{n} \quad \text { for all } n \in \mathbb{N} \text {. }
$$

Taking the orthogonal projection $Q$ of (3.11), we get the estimate

$$
\left\|L u_{n}\right\| \leq t_{n}\left(\left\|w_{n}\right\|+\|h\|\right) \quad \text { for all } n \in \mathbb{N}
$$

According to Lemma 3.2, there is a positive constant $\gamma$ with $\theta<\gamma<3$ such that

$$
\left(w_{n}-h, u_{n}\right) \geq \frac{1}{\gamma}\left\|w_{n}\right\|^{2}-C(h, v) \quad \text { for all } n \in \mathbb{N},
$$


where $C(h, v)$ is a positive constant depending on $h$ and $v$. For all $n \in \mathbb{N}$, we obtain from (3.5), (3.11), and (3.12)

$$
-\frac{1}{3}\left\|L u_{n}\right\|^{2} \leq\left(L u_{n}, u_{n}\right) \leq-t_{n}\left(\frac{1}{\gamma}\left\|w_{n}\right\|^{2}-C(h, v)\right)-\left(1-t_{n}\right)\left(P u_{n}, u_{n}\right)
$$

and hence

$$
\frac{1}{3} t_{n}^{2}\left(\left\|w_{n}\right\|+\|h\|\right)^{2} \geq \frac{1}{3}\left\|L u_{n}\right\|^{2} \geq t_{n}\left(\frac{1}{\gamma}\left\|w_{n}\right\|^{2}-C(h, v)\right)+\left(1-t_{n}\right)\left\|P u_{n}\right\|^{2},
$$

and therefore

$$
\left(t_{n}\left\|w_{n}\right\|\right)\|h\| \geq\left(\frac{1}{\gamma}-\frac{1}{3}\right)\left(t_{n}\left\|w_{n}\right\|\right)^{2}+\left(1-t_{n}\right)\left\|P u_{n}\right\|^{2}-C(h, v)-\|h\|^{2} .
$$

In view of $\gamma<3$, the sets $\left\{t_{n}\left\|w_{n}\right\|\right\}$ and $\left\{\left(1-t_{n}\right)\left\|P u_{n}\right\|^{2}\right\}$ are bounded. Since $\left\{L u_{n}\right\}$ is bounded by (3.12), we see that $\left\{Q u_{n}\right\}$ is also bounded. From $\left\|P u_{n}\right\| \rightarrow \infty$ and $\left(1-t_{n}\right)\left\|P u_{n}\right\| \rightarrow 0$, it follows that $t_{n} \rightarrow 1$. This implies that $\left\{w_{n}\right\}$ is bounded. On the other hand, it follows from (3.9) that $\left\{w_{n}\right\}$ is unbounded, which is a contradiction. Thus, the assertion (3.10) holds for all $t \in[0,1)$ because the reference map $L+P$ is injective with $0 \in(L+P)\left(B_{R}(0) \cap D(L)\right)$. Applying Theorem 2.6 with $G=B_{R}(0)$ and $N_{0}=P$, we conclude that the equation $h \in$ $L u+N u$ has a solution $u$ in $D(L)$. This completes the proof.

Next, we are concerned with the solvability of the given equation (3.4) with nonresonance condition. The single-valued case was discussed in [9].

Theorem 3.4 Suppose that $g: \Omega \times \mathbb{R} \times \mathbb{R} \rightarrow \mathbb{R}$ satisfies the conditions (g1), (g2), and (g3). Let $\tau$ be a positive number such that $-\tau \notin \sigma(L)$ and

$$
\lambda_{N}<a<-\tau<b<\lambda_{N+1}<0,
$$

where $\lambda_{N}$ and $\lambda_{N+1}$ are two consecutive eigenvalues of $L$ and $a, b$ are real numbers. Suppose that there are a positive constant $R$ and a nonnegative function $h_{2} \in H$ such that the following inequalities hold for almost all $(x, t) \in \Omega$ :

$$
\begin{cases}-b s \leq \underline{g}(x, t, s) \leq \bar{g}(x, t, s) \leq-a s & \text { if } \leq \geq R \\ -a s \leq \underline{g}(x, t, s) \leq \bar{g}(x, t, s) \leq-b s & \text { if } \leq-R \\ |g(x, t, s)| \leq h_{2}(x, t) & \text { if }|s|<R .\end{cases}
$$

Then (3.4) has a solution $u$ in $D(L)$ for any $h \in H$.

Proof Let $\tau$ be a positive number such that $-\tau \notin \sigma(L)$ and $a<-\tau<b$. For almost all $(x, t) \in$ $\Omega$ and all $s \in \mathbb{R}$, we have by (3.14)

$$
|w(x, t)-\tau s| \leq \max \{|a+\tau|,|b+\tau|\}|s|+\left|\lambda_{N}\right| R+h_{2}(x, t),
$$

where $g(x, t, s) \leq w(x, t) \leq \bar{g}(x, t, s)$. If we set $\eta_{\tau}=\max \{|a+\tau|,|b+\tau|\}$, then we have by (3.13)

$$
\eta_{\tau}<\operatorname{dist}(-\tau, \sigma(L))
$$


According to (3.15), we can choose a positive constant $\beta$ such that

$$
\|w-\tau u\| \leq \eta_{\tau}\|u\|+\beta \quad \text { for all } u \in H \text { and all } w \in N u .
$$

Now, let $h$ be any element of $H$. We first prove that there is a positive constant $R_{1} \geq R$ such that

$$
0 \notin L u+t(N u-h)+(1-t) \tau u \quad \text { for all } u \in D(L) \text { with }\|u\|=R_{1} \text { and all } t \in(0,1) \text {. }
$$

We argue by contradiction. Assume that there are sequences $\left(u_{n}\right)$ in $D(L)$ with $\left\|u_{n}\right\| \rightarrow \infty$, $\left(t_{n}\right)$ in $(0,1)$ with $t_{n} \rightarrow t$, and $\left(w_{n}\right)$ in $H$ with $w_{n} \in N u_{n}$ such that

$$
L u_{n}+\tau u_{n}+t_{n}\left(w_{n}-\tau u_{n}\right)=t_{n} h \quad \text { for all } n \in \mathbb{N}
$$

Due to $-\tau \notin \sigma(L)$, it is well known that

$$
\left\|(L+\tau I)^{-1}\right\|=\frac{1}{\operatorname{dist}(-\tau, \sigma(L))} .
$$

For all $n \in \mathbb{N}$, we have by (3.16) and (3.18)

$$
\begin{aligned}
\operatorname{dist}(-\tau, \sigma(L))\left\|u_{n}\right\| & \leq\left\|(L+\tau I) u_{n}\right\| \\
& \leq\left\|w_{n}-\tau u_{n}\right\|+\|h\| \\
& \leq \eta_{\tau}\left\|u_{n}\right\|+\|h\|+\beta
\end{aligned}
$$

and hence

$$
\left(\operatorname{dist}(-\tau, \sigma(L))-\eta_{\tau}\right)\left\|u_{n}\right\| \leq\|h\|+\beta,
$$

which is impossible because the left-hand side of (3.19) tends to infinity. Moreover, (3.17) holds for $t=0$ since the linear operator $L+\tau I$ is a reference map. Thus, the boundary condition (3.17) holds for all $t \in[0,1)$. Applying Theorem 2.6 with $G=B_{R_{1}}(0)$ and $N_{0}=\tau I$, the equation $h \in L u+N u$ has a solution $u$ in $D(L)$. This completes the proof.

\section{Competing interests}

The authors declare that they have no competing interests.

\section{Authors' contributions}

I-SK conceived of the study and drafted the manuscript. J-HB and S-JH participated in the coordination. All authors approved the final manuscript.

\section{Acknowledgements}

This work was supported by Sungkyun Research Fund, Sungkyunkwan University, 2014.

Received: 4 September 2015 Accepted: 21 October 2015 Published online: 29 October 2015

\section{References}

1. Brézis, $H$, Nirenberg, L: Characterizations of the ranges of some nonlinear operators and applications to boundary value problems. Ann. Sc. Norm. Super. Pisa, Cl. Sci. (4) 5(2), 225-326 (1978)

2. Brézis, H, Nirenberg, L: Forced vibrations for a nonlinear wave equation. Commun. Pure Appl. Math. 31, 1-30 (1978)

3. Rabinowitz, PH: Free vibrations for a semilinear wave equation. Commun. Pure Appl. Math. 31, 31-68 (1978) 
4. Berkovits, J, Mustonen, V: An application of the topological degree theory for semilinear equations with strongly monotone nonlinearities. Bull. Lond. Math. Soc. 23, 470-476 (1991)

5. Berkovits, J, Mustonen, V: On multiple solutions for a class of semilinear wave equations. Nonlinear Anal. 16(5), 421-434 (1991)

6. Berkovits, J, Mustonen, V: On the existence of multiple solutions for semilinear equations with monotone nonlinearities crossing a finite number of eigenvalues. Nonlinear Anal. 17(5), 399-412 (1991)

7. Mawhin, J, Willem, M: Operators of monotone type and alternative problems with infinite dimensional kernel. In: Recent Advances in Differential Equations (Trieste, 1978), pp. 295-307. Academic Press, New York (1981)

8. Mawhin, J: Nonlinear functional analysis and periodic solutions of semilinear wave equations. In: Laksmikantham, V (ed.) Nonlinear Phenomena in Mathematical Sciences, pp. 671-681. Academic Press, New York (1982)

9. Berkovits, J, Mustonen, V: An extension of Leray-Schauder degree and applications to nonlinear wave equations. Differ. Integral Equ. 3, 945-963 (1990)

10. Berkovits, J, Tienari, M: Topological degree theory for some classes of multis with applications to hyperbolic and elliptic problems involving discontinuous nonlinearities. Dyn. Syst. Appl. 5(1), 1-18 (1996)

11. Chang, KC: The obstacle problem and partial differential equations with discontinuous nonlinearities. Commun. Pure Appl. Math. 3(2), 117-146 (1980)

12. Hofer, H: A multiplicity result for a class of nonlinear problems with applications to a nonlinear wave equation. Nonlinear Anal. 5(1), 1-11 (1981)

\section{Submit your manuscript to a SpringerOpen ${ }^{\ominus}$ journal and benefit from:}

- Convenient online submission

Rigorous peer review

- Immediate publication on acceptance

- Open access: articles freely available online

- High visibility within the field

- Retaining the copyright to your article 